
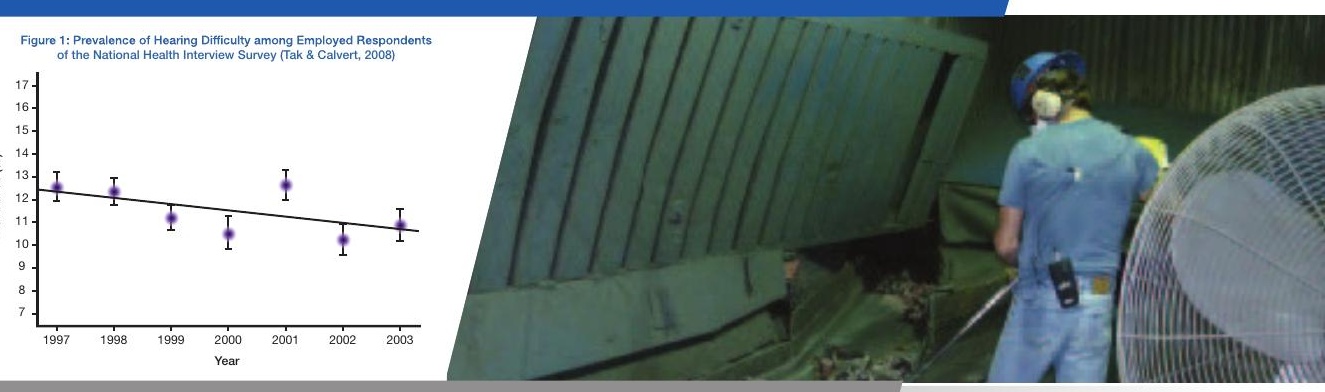

\title{
Occupational Hearing Loss Surveillance
}

Occupational hearing loss (OHL) may occur when workers are exposed to loud noise and/or ototoxic chemicals such as heavy metals, organic solvents, and asphyxiants. Surveillance is vital for preventing OHL. The establishment of prevalence and incidence estimates of hearing loss within industries is made possible through surveillance. It also enables researchers and others to identify high-risk groups, guide prevention and research efforts, and evaluate the success or failure of interventions.

The National Institute for Occupational Safety and Health (NIOSH) initiated the OHL Surveillance Project in 2009 to develop a national surveillance system for OHL. The project uses a novel approach to data collection by partnering with audiometric service providers and others to collect de-identified worker audiograms and related data. This approach allows NIOSH to collect millions of de-identified audiograms from thousands of workplaces across the United States while protecting the identities of workers, companies, and data providers. NIOSH then performs OHL surveillance and research. Findings are available for use by policy makers, researchers, workers, employers, and other stakeholders.

Partnering with new data providers, data collection, statistical analyses, and dissemination of research results are ongoing. Project results indicate workers in mining, construction, and manufacturing have the highest risks for hearing loss, but no industry can be assumed 'safe' from exposures that damage hearing, including noise and ototoxic chemicals.

DHHS (NIOSH) Publication No. 2013-130 (Revised)
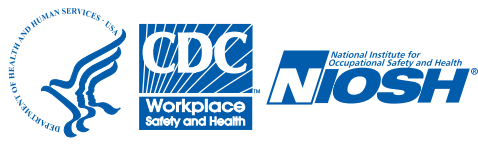

April 2018

\section{Partner With Us}

To learn more about Occupational Hearing Loss Surveillance or to become a partner, visit our Web page at http://www.cdc.gov/niosh/topics/ohl/ or call 513-841-4303. 